\title{
Recombinant Antibodies Derived From Convalescent Whole Blood May Be the Answer to COVID-19
}

\author{
Laalithya Konduru ${ }^{1,2, *}$ \\ ${ }^{1}$ College of Medicine and Public Health, Flinders University, Adelaide, Australia \\ ${ }^{2}$ Public Health Research Unit, Samanjasa Foundation, Chennai, India \\ "Corresponding author: College of Medicine and Public Health, Flinders University, Adelaide, Australia. Email: laalithya@gmail.com \\ Received 2020 May 03; Revised 2020 May 16; Accepted 2020 May 21.
}

Keywords: Emerging New Infection, COVID19, Passive Immunity, Recombinant Antibodies

\section{Dear Editor,}

As of 1st May 2020, the SARS-CoV-2 virus has affected 3,308,555 people all over the world, and caused 234,112 deaths. There are currently no vaccines and no specific therapeutic agents have been shown to be effective against COVID-19 caused by SARS-CoV-2 (1).

In 1894, Emil von Behring treated diphtheria with antisera, and in the process discovered passive immunity. He won the Nobel prize in Medicine in 1901 for this discovery. Since then, antibodies have been used to prevent or treat many infections like Tetanus, Botulism, Hepatitis A, Chickenpox, Rabies, Measles, etc. (2).

Convalescent plasma is plasma obtained from people who have recovered from an infection. It contains antibodies that can confer passive immunity. Convalescent plasma has been used successfully and without complications during past outbreaks of SARS-CoV in 2003 (3), Ebola in 2015 (4), and Influenza A (H1N1) in 2009 (5). Given that a vaccine against SARS-CoV-2 is at least months away, convalescent plasma could be a potential treatment option for COVID-19.

A case-series of 5 critically-ill patients treated with convalescent plasma was reported by Shen et. al. in March 2020. It was reported that viral load reduced in all 5 patients following the treatment, and 3 out of 5 patients were discharged from hospital (6). This study is however limited by the fact that there was no control group, therefore it is not possible to ascertain if these patients could have recovered without the treatment. Continued administration of steroids and antiviral agents after the administration of convalescent plasma confound the results. However, the results make a compelling case for conducting randomised controlled trials of convalescent plasma in treating COVID-19. Passive immunity is more effective as prophylaxis than as therapy. However, prophylactic use of convalescent plasma against COVID-19 is not possible due to the scarce nature of convalescent plasma. A donor weighing $60 \mathrm{~kg}$ may not safely donate more than $690 \mathrm{~mL}$ of plasma twice a week, and the protocol followed by Shen et al. (6) required the transfusion of $400 \mathrm{ml}$ of plasma per recipient.

Instead of relying completely on a finite resource like convalescent plasma, we can use recombinant antibodies. It is possible to manipulate these antibodies in-vitro such that the recombinant antibody has a higher affinity to the antigen than the original antibody (7). Recombinant antibodies have not been previously used to generate vaccines or therapeutics for emergent infections, but they are amenable to such use.

Detailed protocols for the generation of such recombinant antibodies can be found in the literature (8), the discussion of which is outside the scope of this article. Briefly, lymphocytes can be isolated from the peripheral blood of people who have recovered from COVID-19, and having a sufficiently high neutralizing antibody titer. The entire repertoire of antibody genes can then be expressed as a phage display library. SARS-CoV-2 can be grown in an appropriate cell line like VeroE6 or Vero/hSLAM, and the display libraries can be panned against the cell line to select antibodies with the highest affinity. These antibodies can then be cloned and using a high yield expression system, can be isolated from this system. The generation of these monoclonal recombinant antibodies may take a few months. Therefore simultaneously, lymphocytes isolated from convalescing patients must be cultured and immortalised using EBV (9) or CD40L/IL4 (10) and polyclonal antibody cocktail can be isolated from this culture. Such a polyclonal antibody cocktail can be made available in a few weeks. Antibodies thus isolated can be trialled in in-vitro 
studies to assess efficacy in reducing viral load. Antibodies found to be efficacious in-vitro can be trialled in COVID-19 patients to assess therapeutic benefit. In-vivo experiments and clinical trials can also be conducted with these antibodies to assess if they can be used to confer passive immunity against SARS-CoV-2 in non-infected healthy persons.

Though active immunity confers long lasting protection, passive immunity from convalescent plasma and recombinant antibodies isolated from convalescent whole blood confers immediate protection against disease, and in the middle of a raging pandemic like the one we are currently facing, it is just what we need. We should be focusing our energies on tapping its potential.

\section{Footnotes}

Authors' Contribution: Study concept and design: LK. Drafting of the manuscript: LK. Critical revision of the manuscript for important intellectual content: LK.

Conflict of Interests: None.

Funding/Support: None.

\section{References}

1. Wu Z, McGoogan JM. Characteristics of and important lessons from the coronavirus disease 2019(COVID-19) Outbreak in China: Summary of a report of 72314 cases from the chinese center for disease control and prevention. JAMA. 2020. doi: 10.1001/jama.2020.2648. [PubMed: 32091533].

2. Graham BS, Ambrosino DM. History of passive antibody administration for prevention and treatment of infectious diseases. Curr Opin
HIV AIDS. 2015;10(3):129-34. doi: 10.1097/COH.0000000000000154. [PubMed: 25760933]. [PubMed Central: PMC4437582].

3. Cheng Y, Wong R, Soo YO, Wong WS, Lee CK, Ng MH, et al. Use of convalescent plasma therapy in SARS patients in Hong Kong. Eur J Clin Microbiol Infect Dis. 2005;24(1):44-6. doi: 10.1007/s10096-004-1271-9. [PubMed: 15616839]. [PubMed Central: PMC7088355].

4. Arabi Y, Balkhy H, Hajeer AH, Bouchama A, Hayden FG, Al-Omari A, et al. Feasibility, safety, clinical, and laboratory effects of convalescent plasma therapy for patients with Middle East respiratory syndrome coronavirus infection: A study protocol. Springerplus. 2015;4:709. doi: 10.1186/s40064-015-1490-9. [PubMed: 26618098]. [PubMed Central: PMC4653124].

5. Hung IFN, Yuen KY. Hyperimmune IV immunoglobulin treatment of 2009 Influenza A(H1N1): Response. Chest. 2013;144(2):712-3. doi: 10.1378/chest.13-0571.

6. Shen C, Wang Z, Zhao F, Yang Y, Li J, Yuan J, et al. Treatment of 5 critically Ill patients With COVID-19 with convalescent plasma.JAMA. 2020 doi: 10.1001/jama.2020.4783. [PubMed: 32219428]. [PubMed Central: PMC7101507].

7. Smith KA, Nelson PN, Warren P, Astley SJ, Murray PG, Greenman J. Demystified...recombinant antibodies. J Clin Pathol. 2004;57(9):912-7. doi: 10.1136/jcp.2003.014407. [PubMed: 15333649]. [PubMed Central: PMC1770420].

8. Cai X, Garen A. Anti-melanoma antibodies from melanoma patients immunized with genetically modified autologous tumor cells: Selection of specific antibodies from single-chain Fv fusion phage libraries. Proc Natl Acad Sci U S A. 1995;92(14):6537-41. doi: 10.1073/pnas.92.14.6537. [PubMed: 7604028]. [PubMed Central: PMC41553].

9. Fraussen J, Vrolix K, Martinez-Martinez P, Losen M, Meulemans E, De Baets $\mathrm{MH}$, et al. A novel method for making human monoclonal antibodies. J Autoimmun. 2010;35(2):130-4. doi: 10.1016/j.jaut.2010.05.001 [PubMed: 20732843]. [PubMed Central: PMC7126289].

10. Wiesner M, Zentz C, Mayr C, Wimmer R, Hammerschmidt W, Zeidler $\mathrm{R}$, et al. Conditional immortalization of human B cells by CD40 lig ation. PLoS One. 2008;3(1). e1464. doi: 10.1371/journal.pone.0001464. [PubMed: 18213373]. [PubMed Central: PMC2180193]. 